\title{
EchoGéo
}

46 | 2018

Varia

\section{Invisibilité des risques sanitaires et ambivalences des héritages industriels en Seine-Saint-Denis}

Axelle Croisé, Alexis Sierra et Benjamin Lysaniuk

\section{OpenEdition}

Journals

Édition électronique

URL : https://journals.openedition.org/echogeo/16180

DOI : 10.4000/echogeo.16180

ISSN : 1963-1197

Éditeur

Pôle de recherche pour l'organisation et la diffusion de l'information géographique (CNRS UMR 8586)

Référence électronique

Axelle Croisé, Alexis Sierra et Benjamin Lysaniuk, «Invisibilité des risques sanitaires et ambivalences des héritages industriels en Seine-Saint-Denis », EchoGéo [En ligne], 46 | 2018, mis en ligne le 31 décembre 2018, consulté le 31 juillet 2021. URL : http://journals.openedition.org/echogeo/16180 ; DOI : https://doi.org/10.4000/echogeo.16180

Ce document a été généré automatiquement le 31 juillet 2021.

EchoGéo est mis à disposition selon les termes de la licence Creative Commons Attribution - Pas d'Utilisation Commerciale - Pas de Modification 4.0 International (CC BY-NC-ND) 


\title{
Invisibilité des risques sanitaires et ambivalences des héritages industriels en Seine-Saint-Denis
}

\author{
Axelle Croisé, Alexis Sierra et Benjamin Lysaniuk
}

1 Le territoire correspondant à l'actuel département de la Seine-Saint-Denis fut le théâtre d'une industrialisation massive au cours des Révolutions Industrielles (Vieillard-Baron, 2011). L'implantation d'industries dites «lourdes" (minière, électrique, chimique, métallurgique ou mécanique) s'accompagna du développement d'un maillage de petites et de très petites industries sous-traitantes. Ces dernières s'établirent à proximité de leurs commanditaires, d'abord dans les communes limitrophes de Paris et le long des nouveaux axes de transport qui se développèrent au XIX siècle : les canaux de SaintDenis et de l'Ourcq et les lignes ferroviaires reliant Paris au Nord (Paris-Lille) et à l'Est (Paris-Metz) du pays. Les «nuisances», terme usité pour évoquer la pollution perceptible par les sens et en particulier par l'odorat et la vue (Massard-Guilbaud, 2010), générées par ces activités furent telles (Guillerme et al., 2004) qu'elles devinrent une caractéristique intrinsèque de cet espace industrialisé (Fourcaut, 2007).

2 Outre une pollution immédiate et encore effective des sols ${ }^{1}$ engendrée par le « débordement » (Letté, 2011) des nuisances hors des murs des usines, l'exposition des travailleurs et des riverains à des produits et process industriels néfastes peut affecter leur santé parfois plusieurs décennies après leur exposition (Hill, 1965). Ainsi, le décès d'un Aulnaysien des suites d'un mésothéliome pleural ${ }^{2}$ fut finalement imputé au Comptoir des Minéraux et de Matières Premières (CMMP). Cette usine broyait de l'amiante (1938-1975) à l'époque où la victime était scolarisée dans une école voisine. Le diagnostic fut établi 20 ans après l'arrêt officiel de cette activité (1995) et quatre ans après la fermeture de l'usine. Le lien entre l'environnement des habitants et des travailleurs et le déclenchement de la maladie est ainsi difficile à établir du fait de ce temps de latence, car, au moment où le citadin est diagnostiqué malade, l'environnement urbain a profondément changé. L'imperceptibilité de certaines pollutions pathogènes ${ }^{3}$ (inodores, incolores, souterraines) combinée à cette apparition 
différée dans le temps et dans l'espace des pathologies accroît la difficulté à établir ce lien pour les victimes et les experts (Gollac et Volkoff, 2007). Ces pathologies ne deviennent souvent "visibles" que lors de mobilisations citoyennes ou d'experts déclenchées par la découverte d'une pollution et/ou d'un agrégat de malades (souvent atteints de cancers) autour d'un site en activité ou anciennement actif (Calvez, 2009). Notre démarche consiste alors à analyser les risques de cancers professionnels par les lieux, c'est-à-dire par les sites industriels susceptibles d'être à l'origine de la maladie. Le risque étant par définition une réalité abstraite, l'espace-à-risque en est la face concrète permettant de le "gérer " (Sierra, 2000), c'est-à-dire de le saisir et de le rendre visible pour le traiter. Le lieu, concret, situé, alimente la controverse qui résulte de l'attribution de multiples représentations, parfois contradictoires, à ce même espace (Retaillé, 2011). L'identification du lieu générateur de l'aléa est un moyen de réduire l'incertitude du lien causal entre activité professionnelle et pathologie et devient ainsi l'enjeu du débat. En associant le danger à un lieu, matériel, morphologiquement inscrit et identifié sur le territoire, les acteurs de la controverse le rendent visible alors que l'activité génératrice de la pollution a pu disparaître ou reste dissimulée entre les murs de l'entreprise. Nous faisons ainsi l'hypothèse que c'est l'identification d'anciens sites industriels comme "pathogènes» qui peut rendre visibles l'exposition, les pathologies induites et 医 par extension 原 les malades. La relation au territoire est d'autant plus prégnante dans l'identification et la reconnaissance de tels «lieux-à-risque », qu'elle concerne un espace particulièrement industrialisé (Osadtchy, 2015) subissant des modifications économique, politique, sociale et urbaine majeures du fait de son importance stratégique dans la géographie francilienne. Or, malgré le riche passé industriel et la profonde empreinte laissée par cette activité sur le territoire départemental, seuls quelques sites semblent soumis à controverses (tableau 1). Le tableau ne dépeint probablement pas une liste exhaustive de ces lieux, mais présentent les plus médiatiques et donc les plus visibles. La relation à ce passé et à ces sites industriels apparait ainsi ambivalente.

Tableau 1 - Les lieux d'activité soumis à controverse en Seine-Saint-Denis

\begin{tabular}{|c|c|c|c|}
\hline Localisation & Sites et/ou activités & Mobilisations & Pathologies \\
\hline $\begin{array}{l}\text { Aulnay-sous- } \\
\text { Bois }\end{array}$ & $\begin{array}{lrr}\text { Comptoir } & \text { des } \\
\text { Minéraux } & \text { et des } \\
\text { Matières } & \text { Premières : } \\
\text { broyage } & \text { d'amiante } \\
(1938-1975) & \text { (fermé } & \text { en } \\
1999) & & \end{array}$ & $\begin{array}{l}\text { Mobilisation de riverains suite à } \\
\text { la découverte du mésothéliome } \\
\text { d'un habitant (1995) puis appui } \\
\text { scientifique (rapport de l'Institut } \\
\text { de Veille Sanitaire et } \\
\text { Groupement d'Intérêt } \\
\text { Scientifique sur les Cancers } \\
\text { d'Origine Professionnelle 93) }\end{array}$ & $\begin{array}{l}234 \text { malades } \\
\text { (mésothéliomes, } \\
\text { cancers broncho- } \\
\text { pulmonaires, } \\
\text { plaques pleurales...) }\end{array}$ \\
\hline $\begin{array}{l}\text { Les } \\
\text { Pavillons- } \\
\text { sous-Bois }\end{array}$ & $\begin{array}{l}\text { Dépôts d'ordures } \\
\text { ménagères (1914-1932 } \\
\text { puis 1939-1959) } \\
\text { Remblaiements de } \\
\text { nature indéterminée } \\
(1950-1959)\end{array}$ & $\begin{array}{l}\text { Mobilisation de riverains suite à } \\
\text { un contrôle de pollution des sols } \\
(2002)\end{array}$ & $\begin{array}{l}\text { Suspicion de cas de } \\
\text { saturnisme }\end{array}$ \\
\hline
\end{tabular}




\begin{tabular}{|c|c|c|c|}
\hline Montreuil & $\begin{array}{l}\text { Société Nouvelle } \\
\text { d'Eugénisation des } \\
\text { Métaux : traitement } \\
\text { chimiques des métaux } \\
\text { pour l'aéronautique (en } \\
\text { activité) }\end{array}$ & $\begin{array}{l}\text { Mobilisation de riverains (2005) } \\
\text { puis de parents d'élèves à la } \\
\text { suite de cas de leucémies }\end{array}$ & $\begin{array}{l}3 \text { cas de leucémies } \\
\text { rares chez les enfants } \\
\text { dans la même rue (sur } \\
15 \text { ans) }\end{array}$ \\
\hline Vaujours & $\begin{array}{l}\text { Commissariat de } \\
\text { l'Énergie Atomique : } \\
\text { essais nucléaires sous- } \\
\text { critiques tirs " froids ") } \\
(1955-1997) \\
\text { BP Placo : fabrication de } \\
\text { plâtre (terrain acheté en } \\
\text { 2010) (en activité) }\end{array}$ & $\begin{array}{l}\text { Mobilisation de riverains suite à } \\
\text { un rapport mettant en avant la } \\
\text { pollution du site (2002) }\end{array}$ & $\begin{array}{l}\text { Cas de cancers } \\
\text { (tyroïde) }\end{array}$ \\
\hline Romainville & $\begin{array}{l}\text { UMM : fabrication de } \\
\text { vannes (1929-1977) } \\
\text { Wipelec : traitements } \\
\text { des métaux et des } \\
\text { matières plastiques } \\
(2002-2003)\end{array}$ & $\begin{array}{l}\text { Mobilisation de riverains suite à } \\
\text { un rapport de l'ADEME sur la } \\
\text { pollution des sols (2016) }\end{array}$ & $\begin{array}{l}23 \text { cancers dont } \\
21 \text { décès. } \\
\text { Suspicion de cas de } \\
\text { saturnisme }\end{array}$ \\
\hline Saint-Ouen & $\begin{array}{l}\text { Wonder : fabrication de } \\
\text { piles (1918-1986) }\end{array}$ & $\begin{array}{l}\text { Mobilisation de parents d'élèves } \\
\text { suite au démarrage d'un } \\
\text { chantier }\end{array}$ & $\begin{array}{l}\text { Crainte d'impacts } \\
\text { sanitaires pour les } \\
\text { adolescents du lycée } \\
\text { Joliot-Curie }\end{array}$ \\
\hline
\end{tabular}

Source : Redon F, 2018, p. 10. Auteur : Liste complétée par A. Croisé, décembre 2018.

3 Notre hypothèse est que le "recyclage urbain " (Veschambre, 2005) des anciens sites industriels, à travers les opérations de reconversion, de rénovation et de réhabilitation ${ }^{4}$, contribue à l'invisibilité de la pathogénicité des sites, faisant de l'ensemble du département un terrain d'étude privilégié de ce type d'évolution.

De fait, il n'existe pas de liste exhaustive de sites industriels. Partant de ce constat, nous avons choisi de nous intéresser spécifiquement aux sites médiatisés, c'est-à-dire ceux dont le passé est véhiculé, mais qui ne sont pas pour autant reconnus comme potentiellement pathogènes. Nous nous focaliserons sur les transformations qu'ils ont pu subir afin de proposer une typologie des formes de recyclage urbain du point de vue de leur incidence sur la représentation du risque pathogène.

\section{Quelles « traces » du passé industriel en Seine-Saint- Denis ?}

5 Établir une liste des sites industriels est une gageure. La phase de désindustrialisation et de reconversion urbaine ont provoqué la disparition de nombreuses traces du passage de l'industrie dans le département. Cette disparition est à la fois matérielle, avec la destruction des archives d'entreprise et des bâtiments industriels, et 
informative, le savoir diffusé se limitant à un nombre restreint de sites classés au titre d'un patrimoine dit vernaculaire.

\section{L'effondrement d'un secteur d'activité}

6 La crise du secteur industriel s'abat dès les années 1960 sur la Plaine-Saint-Denis, regroupant les communes d'Aubervilliers, de Saint-Ouen et le Sud de Saint-Denis. À l'échelle départementale ce sont 4000 emplois industriels qui disparaissent tous les ans entre 1968 et 1972. Ces pertes sont en partie compensées par le développement de nouvelles zones d'activité au Blanc-Mesnil (ZA du Coudray), à Aulnay-sous-Bois (ZI de La Garenne) et à Bagnolet (ZI de La Noue) (Lysaniuk et al., 2015). Mais l'hémorragie n'est pas pour autant stoppée. Ainsi, $70 \%$ des emplois industriels existants disparaissent dans le département entre 1982 et 2010, faisant passer sa part dans l'emploi de $40 \%$ à moins de $10 \%$ (Direccte île-de-France, 2011). Cette crise économique et sociale se ressent dans la structure même du paysage séquano-dionysien. Les infrastructures industrielles inoccupées sont laissées en friche et deviennent la marque visible de la crise.

Un glissement progressif s'opère alors vers le secteur tertiaire. Celui-ci est impulsé conjointement par les communes et l'État, quand en 1993, l'État choisit Saint-Denis et le site des anciens gazomètres de la société du Gaz de France pour construire le Stade de France. Le passé industriel disparaît ainsi pour laisser place au $4^{\mathrm{e}}$ pôle tertiaire d'île-deFrance (Lebeau, 2014). La tertiarisation du territoire se propage à l'ensemble du département. Entre 1982 et 2010 près de 156000 emplois ont été créés faisant passer la part du tertiaire dans l'emploi départemental de $48 \%$ à $80 \%$ (Direccte Île-de-France, ibid.). Ainsi, en lieu et place des anciennes industries, s'installent des bureaux dont de nombreux sièges d'entreprises (SCNCF, Orange, Siemens...), résultats des opérations de reconversions urbaines.

Cette évolution de l'emploi et du paysage dans le département n'est pas non plus sans conséquences pour les Séquano-dionysiens. Depuis la deuxième moitié du XIX ${ }^{e}$ siècle, cette population est étroitement liée au monde ouvrier: les phénomènes combinés d'industrialisation intensive du territoire, de mise en place des voies de chemin de fer et d'attractivité foncière (de vastes espaces disponibles et peu cher), largement exploitée par les promoteurs immobiliers, participèrent à l'installation des ouvriers dans le département. Profondément associé au monde industriel, le département devint aussi électoralement une «banlieue rouge ». Cependant, la désindustrialisation change la donne. La formation et l'expérience professionnelle des Séquano-dionysiens ne correspondent plus aux emplois disponibles. Dès 1982, le taux de chômage y est supérieur au taux régional, dépassant celui de la France métropolitaine dès 1985 et dépassant les $10 \%$ en 1993. Ainsi, en 2015, ils ne sont plus que 130000 ouvriers à avoir un emploi alors qu'ils étaient 240000 en 1968, soit un taux d'évolution négatif de près de $45 \%{ }^{5}$. Face à de tels chiffres, nous pouvons penser que la disparition des sites industriels et de leurs travailleurs tend à étioler la mémoire des activités passées et des risques qui y sont associés, restreignant la capacité des populations actuelles à identifier la pathogénicité des sites en lien avec leurs activités passées et à se mobiliser autour de l'un d'entre eux. 


\section{Réagir face aux destructions : le choix de la patrimonialisation}

9 La cessation brutale des activités en Seine-Saint-Denis provoque la mise en friche, plus ou moins longue (de quelques mois à quelques années), des installations (Edelblutte, 2010). La friche industrielle, urbaine, devient alors le symbole de la crise économique et sociale avant de devenir un espace vacant et « ressource » (Ambrosino et Andres, 2008) pour un futur aménagement, et ce ${ }_{20}$ en particulier ${ }_{20}$ en banlieue parisienne, dans un espace où la disponibilité foncière est recherchée. Les friches sont alors réinvesties dans les années 1980 afin de maintenir l'emploi puis dans les années 2000 dans une tentative de requalification urbaine (Furio, 2012). Cela se traduit aussi bien par la destruction que par le maintien d'une partie ou de l'ensemble des anciens bâtiments industriels.

La destruction des bâtiments industriels est une action forte, symbolique, souvent liée à une perception négative de l'activité industrielle qui émerge au milieu du $\mathrm{XX}^{\mathrm{e}}$ siècle avec le développement d'une conscience environnementale (Edelblutte, 2008). Détruire permet de faire table rase d'un passé douloureux, associé à l'échec d'un secteur d'activité. En effet, les anciens bâtiments industriels ne sont pas uniquement des lieux "dont on se souvient", ils sont des lieux « où l'on se souvient» (Sgard, 2007). Ils représentent une fenêtre ouverte sur un passé révolu qui, par son ancrage territorial, lui permet d'exister, de persister et de transposer son histoire dans le temps et dans l'espace (ibid.). C'est par la disparition de la pierre que l'histoire et la mémoire (individuelle et collective) de l'activité et de ses conséquences sanitaires sont atteintes (Veschambre, 2005). Inversement, les sites conservés sont susceptibles d'être considérés comme les objets d'un patrimoine vernaculaire séquano-dionysien s'ils sont choisis et reconnus comme support d'un lien tangible (qu'il est néanmoins nécessaire de reconstruire) avec un passé révolu (Davallon, 2002). Objet d'étude de choix, les sites patrimonialisés sont nécessairement le reflet déformé d'une réalité qui n'est plus. Le patrimoine est alors intrinsèquement lié à la mémoire. Audrey Tuaillon-Demésy (2013) remarque que « mémoire et patrimoine sont compris ensemble : il s'agit d'acquérir des compétences liées à un passé indéfini, de les conserver puis de les transmettre ». La différence entre les deux processus, outre celle d'un héritage « subi » de la mémoire et celui d'un héritage "construit» par le patrimoine, serait que le patrimoine, une fois institué, demeure relativement «stable» au contraire de la mémoire collective «variable» (Dalbavie, 2012), particulièrement quand la référence ouvrière a été profondément affectée. Nous supposons donc que le patrimoine vernaculaire lié au territoire constitue le symbole et le média d'une histoire industrielle telle qu'elle est aujourd'hui accessible aux Séquano-dionysiens, générant une mémoire patrimonialisée et partagée de l'industrie du département.

11 De fait, les destructions sont remises en question dès les années 1970 en France (Del Biondo et Edelblutte, 2016) : les pouvoirs publics et les habitants luttent, en réaction, pour conserver les marques de l'industrie (Di Méo, 2007). Cela se traduit par une politique de patrimonialisation à Saint-Denis, renforcée dans les années 1990 par la réhabilitation du centre-ville (Djament-Tran, 2014). Ces sites ont d'autant plus de valeur qu'ils n'entrent guère en concurrence avec le patrimoine traditionnel (religieux) au sein du département. Les destructions ne sont plus systématiques, néanmoins, les sites industriels doivent, pour être conservés, avoir une valeur symbolique, un intérêt (culturel, économique, social), une valeur esthétique (architecturale pour les 
bâtiments) et être viables économiquement sur le temps long (Siméon, 2014). Ainsi, les gazomètres de la société du Gaz de France sont détruits à Saint-Denis comme partout en France (Real, 2015). Les locaux d'Idéal Standard sont, quant à eux, conservés à Aulnay-sous-Bois, car le rapport coût-bénéfice penche pour la réutilisation des infrastructures (Furio, 2012). À l'échelle du département, le Conseil de l'Architecture de l'Urbanisme et de l'Environnement du département (CAUE 93), instauré en 1981, produit des «inventaires du patrimoine industriel» pour huit communes (Aubervilliers, Bagnolet, La Courneuve, Montreuil, Pantin, Romainville, Saint-Denis, Saint-Ouen) ainsi que pour le quartier de la Plaine Saint-Denis. Son action vise à recenser les anciens bâtiments industriels encore existants et à sensibiliser l'opinion publique à la conservation des traces de ce passé. Néanmoins, le CAUE 93 ne semble s'intéresser activement au patrimoine industriel que vingt ans après les premières classifications au patrimoine de l'UNESCO de sites industriels (Siméon, 2014). Le préambule des inventaires dénonce les bouleversements architecturaux subis en SeineSaint-Denis ${ }^{6}$, le désintérêt originel des pouvoirs publics pour les bâtiments en friche conduisant à la disparition du patrimoine industriel (inventaire de Saint-Ouen) et l'intérêt de constituer une mémoire de ce passép . Cependant, cette action valorise essentiellement l'architecture et non l'activité qui lui est associée et peut être potentiellement pathogène. Celle-ci n'est présentée que de façon sommaire, se résumant à une dénomination générale (type "fabrique de peinture et de vernis", "métallurgie ", etc.).

De surcroît, les inventaires ne présentent qu'un choix restreint de sites, sur un panel potentiel beaucoup plus large (tableau 2). Les critères de sélection varient d'un inventaire à un autre et ne sont jamais clairement explicités. Ainsi, les inventaires de Saint-Denis et Romainville ne présentent une photographie exhaustive du tissu industriel que "jusqu'en 1950 ». Par la suite ils indiquent opérer "des choix » sans avoir de critères ni précis ni harmonisés. Certains sites industriels sont intégrés pour leur esthétisme (Saint-Denis, La Plaine Saint-Denis), d'autres sont choisis pour leur date de construction «et non pas en fonction des critères qualitatifs (esthétiques) ou quantitatifs (taille des bâtiments, nombre d'ouvriers...) »(Saint-Ouen, Aubervilliers, Montreuil, La Courneuve). Ceux de Romainville le sont en fonction de critères " historiques » et "esthétiques» enfin les sites de Pantin sont choisis en fonction de «l'intérêt du site».

Tableau 2 - Les inventaires du patrimoine industriel réalisés par le CAUE 93 en Seine-Saint-Denis

\begin{tabular}{|l|l|l|l|}
\hline Commune & Sites trouvés & Sites sélectionnés & Année \\
\hline Aubervilliers & 190 & $81(56:$ Aubervilliers et $25:$ La Courneuve $)$ & 1993 \\
\hline Bagnolet & Environ 200 & 28 & 2004 \\
\hline La Courneuve & 190 & $81(56:$ Aubervilliers et $25:$ La Courneuve $)$ & 1993 \\
\hline La Plaine-Saint-Denis & 94 & 35 & 1993 \\
\hline Montreuil & 177 & 68 & 1991 \\
\hline Pantin & Inconnu & 57 & 1997 \\
\hline
\end{tabular}




\begin{tabular}{|l|l|l|l|}
\hline Romainville & 38 & 22 & 1994 \\
\hline Saint-Denis & Inconnu & 38 & 1988 \\
\hline Saint-Ouen & 144 & 37 & 1994 \\
\hline
\end{tabular}

Source : CAUE 93, consultation de l'ensemble des inventaires du patrimoine. Auteur : A. Croisé, octobre 2017 - janvier 2018

13 En tentant de préserver le passé industriel du département, le CAUE 93 ne met en lumière qu'une partie de ce passé, invisibilisant de fait les sites non classés comme si aucune mémoire n'en était conservée.

\section{L'impossible reconstitution du passé industriel : le prisme des données administratives}

14 Il semble vain d'établir une liste de sites industriels, en activité ou non, en partant du paysage actuel. Les mutations subies dans le département en témoignent, à l'image de la Plaine Saint-Denis, tertiarisée alors qu'elle a été l'espace industriel le plus dense du département, de France et le second d'Europe (Vieillard-Baron, 2011).

Cependant, des listes d'anciens sites d'activités existent à l'échelle du département. Celle qui semble la plus exhaustive demeure la Bases de données des Anciens Sites industriels et Activités de Services (BASIAS). Instituée dans les années 1990, elle tire ses données de l'Inventaire Historique Régional (IHR) opéré par chaque département et supervisé à l'échelle régionale. Elle affiche comme objectifs de "recenser" et " conserver la mémoire de ces sites" tout comme celui de «fournir des informations utiles aux acteurs de l'urbanisme, du foncier et de la protection de l'environnement " sans qu'elle "ne préjuge d'une éventuelle pollution à son endroit». Ainsi, BASIAS comprend une liste de 5375 sites établis en Seine-Saint-Denis de 1790 à nos jours. Néanmoins, l'étude des métadonnées nous révèle ses limites comme outil permettant de développer une mémoire des conditions de travail. À l'échelle de la Seine-SaintDenis, ne sont sélectionnés que les sites connus de l'administration et classés en catégorie I et II par le Service de l'Environnement Industriel (SEI) qui considère trois catégories d'activité. Les produits utilisés et déclarés auxquels pouvaient être exposés les travailleurs sont intégrés à la base sans toutefois faire de lien avec leurs éventuelles conséquences sanitaires ou environnementales. Sans indication sur l'activité réelle de travail menée au sein des sites, la base néglige des expositions probables à des produits et process néfastes subies par les travailleurs.

En revanche, la base de données constituée par le Groupement d'Intérêt Scientifique sur les Cancers d'Origine Professionnelle en Seine-Saint-Denis (GISCOP 93) fait le lien entre des pathologies cancéreuses et des expositions à des cancérogènes subies par des travailleurs sur leur lieu de travail et relative à leur activité réelle de travail. Loin d'être exhaustive, elle recense 352 lieux (géolocalisés) ayant exposé des travailleurs à des cancérogènes en Seine-Saint-Denis, sur un panel possible de plus de 5000 sites identifiés par la base BASIAS (illustration 1). Or, sur cet échantillon restreint, le GISCOP 93 répertorie plus d'une centaine de sites exposant à l'amiante alors que BASIAS n'en recense que 17 (Lysaniuk et al., 2015). Cet écart s'explique par au moins deux raisons : certains sites d'activité non enregistrés par l'administration sont connus 
du GISCOP 93 et la fibre d'amiante peut être présente dans l'infrastructure même du bâtiment ou dans diverses préparations (par exemple des colles, des enduits...), informations non prises en compte par BASIAS.

Illustration 1 - Géolocalisation des sites répertoriés par BASIAS et le GISCOP 93

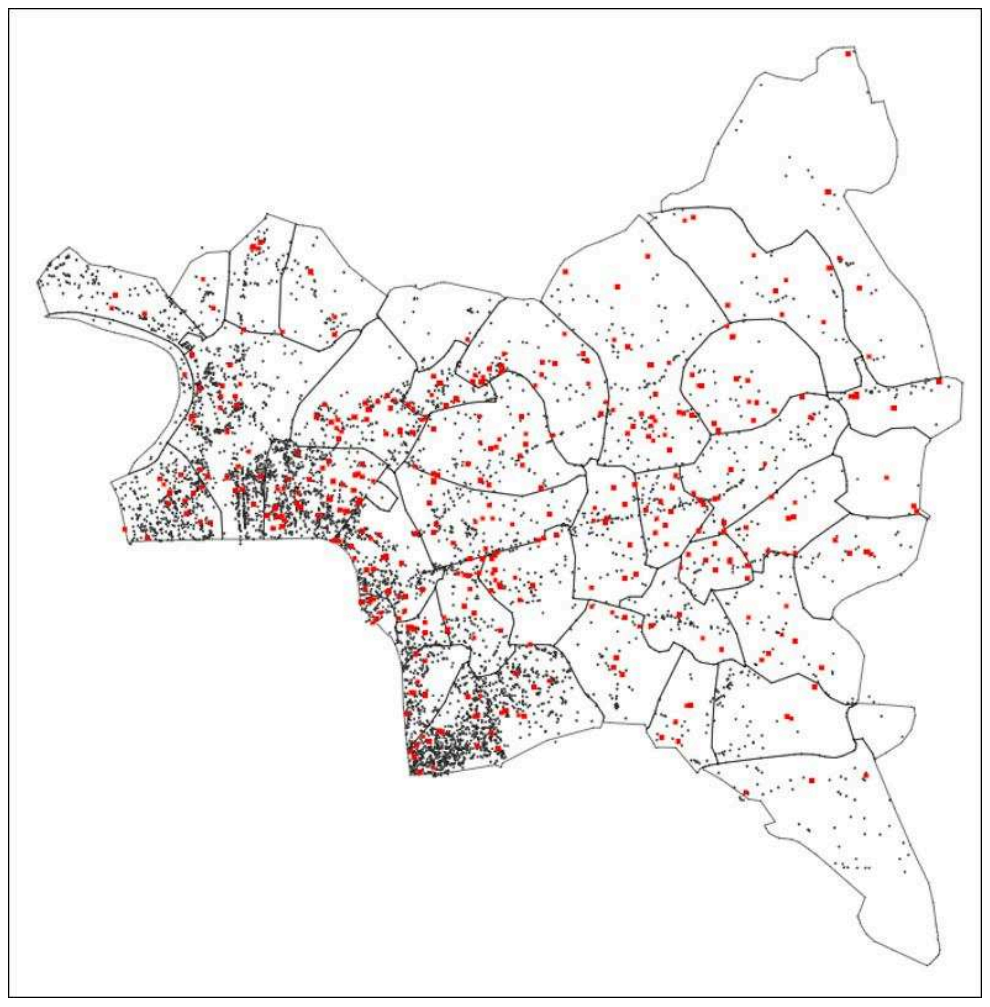

Source : BASIAS, GISCOP. Auteur : A. Croisé, mars 2018

Les autres bases de données censées répertorier les lieux d'activité et d'éventuelles pollutions 㡠 comme la Base de données sur les sites et sols pollués (BASOL), le registre des émissions polluantes (IREP) ou celles sur les Secteurs d'Information sur les Sols (SIS) 医 dépendent, elles aussi, de l'enregistrement fait en préfecture.

Enfin, l'absence de liste exhaustive des sites émetteurs de polluants potentiellement dangereux pour la santé s'explique également par l'absence de cadre réglementant la conservation des archives privées. Rien n'oblige en effet les entreprises à transmettre leurs archives. Légalement, les services compétents (archives communale, départementale, régionale ou nationale) peuvent récupérer les documents des entreprises en cessation d'activité, mais cette législation n'a été mise en place que tardivement et n'est que très rarement appliquée ${ }^{8}$. Ainsi, et ce même si le bâtiment existe toujours, il peut être extrêmement difficile de savoir quelles activités et/ou quelles entreprises, il a hébergées.

\section{Quelle approche mettre en place ?}

Face à la non-exhaustivité des bases de données déjà constituées et à l'absence de critères communs de sélection des sites, nous avons pris le parti de constituer notre propre base de sites. Partant du postulat que les communes sont les plus au fait de leur histoire industrielle, nous nous sommes intéressés aux sites web municipaux. Toutes les 
communes du département possèdent en effet leur propre plateforme numérique, qui rend compte de la valorisation d'anciens sites industriels à des fins d'identité territoriale et/ou à des fins touristiques et pédagogiques 9 . Nous avons donc systématiquement répertorié, sur les sites web des communes ${ }^{10}$, la mention d'une industrie ou d'une activité industrielle s'étant établie dans le département, et ce, jusqu'au début de la désindustrialisation. Les mentions d'un même espace ont été systématiquement préservées puisqu'un même lieu a pu accueillir successivement, voire concomitamment, plusieurs entreprises. Sur le total ainsi recensé, 94 références renvoient à des industries qui ont totalement disparu ou dont ne persistent qu'un ou quelques éléments dans le paysage (de la machine au bâtiment entier), indiquant que le processus mémoriel peut être engagé même en l'absence de trace sur le paysage. Ce qu'éclaire cette approche est surtout que les municipalités valorisent différemment leur passé industriel et, ce faisant, peuvent inégalement contribuer à l'invisibilisation des risques sanitaires qui lui sont liés. Les établissements recensés se trouvent dispersés sur le territoire, mais certaines communes communiquent beaucoup plus sur leur passé que d'autres (illustration 2). À Epinay-sur-Seine, 28 anciens sites industriels sont évoqués alors que la commune est historiquement beaucoup moins industrialisée que celles de la Plaine Saint-Denis (seulement 35 sites). La présence d'un "parcours historique " centré sur le patrimoine industriel de la commune explique le nombre important de sites référencés. La plupart des autres communes se focalisent sur quelques sites comme Guitel (fabrique de roulement à billes) au Pré-Saint-Gervais, la fabrique d'allumettes à Aubervilliers, Westinghouse (fabrique de freins) et Kodak (fabrique de films) à Sevran et la carrière de gypse à Gagny qui sont cités à plusieurs reprises. Ainsi, la réhabilitation et/ou la destruction des bâtiments industriels participeraient à la mise en mémoire d'une histoire de l'industrie séquano-dionysienne (Real, 2015) qu'il s'agirait de comparer avec la pathogénicité effective identifiée pour les mêmes sites par le GISCOP 93, révélant l'écart entre la communication et la nocivité réelle de ces derniers. 
Illustration 2 - Géolocalisation et occurrences des anciens sites industriels médiatisés par les communes

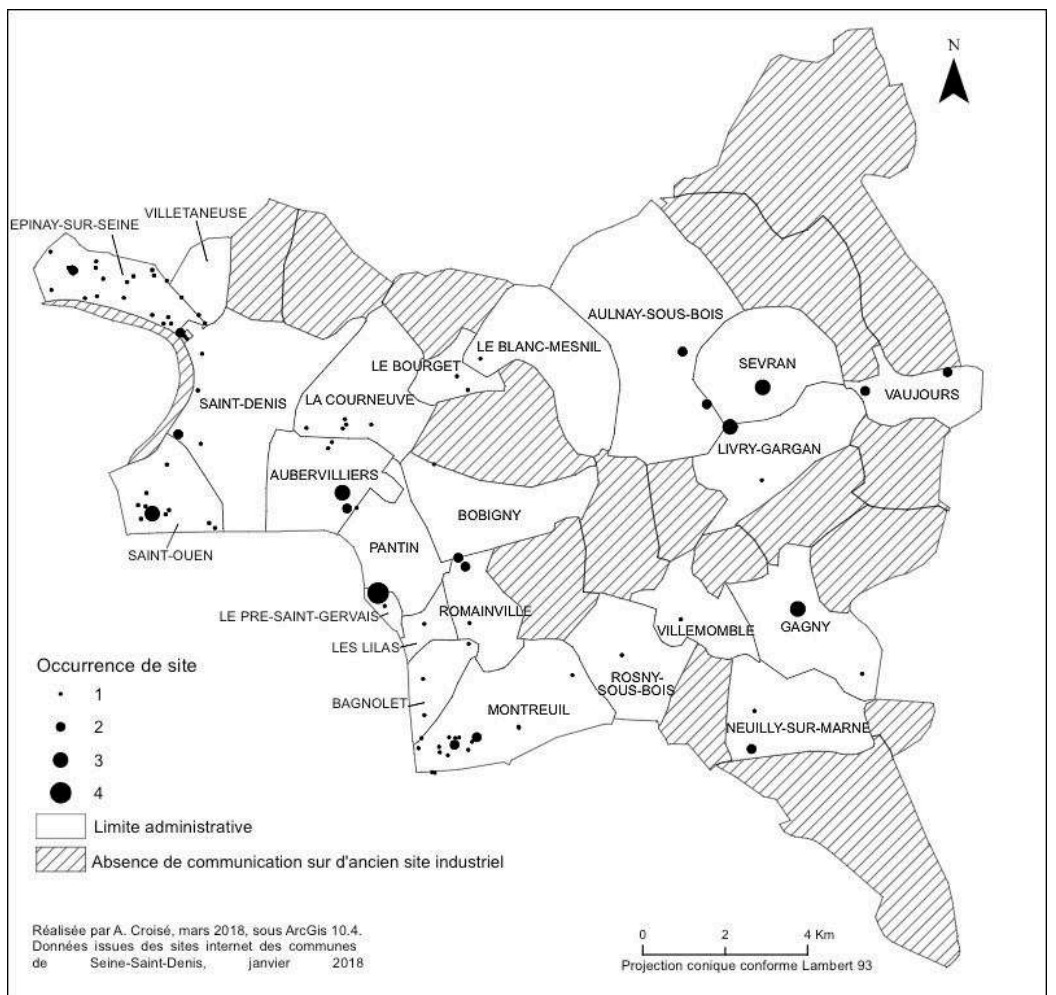

Source : Site web des 40 communes de la Seine-Saint-Denis. Auteur : A. Croisé, janvier 2018

Là où le passé industriel est médiatisé, se pose alors la question du contenu de cette transmission. Puisque ce qui devient «patrimoine» résulte de choix (politiques, économiques, esthétiques, symboliques) (Siméon, 2014), le bâtiment perd une partie de ses caractéristiques intrinsèques et ne garde que ce "qui à valeur à être transmis ". Nous l'abordons alors ici comme un "lieu de mémoire" (Nora, 1984), et comme possible facteur de visibilité d'un risque (celui de perdre sa santé). Néanmoins, la conservation de la pierre seule ne présage pas nécessairement de la conservation et de la transmission de l'histoire industrielle (Furio, 2012), c'est par le discours que celle-ci est transmise pour devenir une mémoire partagée. Ce discours découle de processus mémoriels de sélection et d'oubli, mais aussi d'une forme de travail sur la mémoire, partie prenante d'une idéologie de l'histoire industrielle (ici, une industrie forte, basée sur un savoir-faire) (Ricœur, 2000) qu'il est important d'étudier. Notre hypothèse est que ces deux processus combinés (celui du choix des établissements conservés et du discours qui est véhiculé) participent d'une image valorisante de l'industrie passée qui fait obstacle à la reconnaissance de leur caractère néfaste pour la santé.

\section{L'aseptisation des anciens sites industriels}

Pour comprendre l'intrication des facteurs entre transformations urbaines et invisibilisation du caractère pathogène, nous avons procédé à une enquête de terrain. Nous avons conduit une démarche d'observation directe, principalement de la rue, des anciens sites industriels (listés dans notre inventaire webographique) afin d'observer leurs transformations architecturales et leur intégration (visuelle et économique) dans 
leur environnement actuel. Cette observation directe s'est faite en deux temps, en solitaire et au sein des visites guidées organisées par le département ou les mairies des communes concernées. L'observation est faite en parallèle d'une analyse textuelle de la communication effectuée à la fois sur les plateformes web des communes et sur les sites eux-mêmes à travers les panneaux d'information. Nous souhaitons, à travers ces deux démarches, confronter l'aménagement actuel des sites (comprenant l'architecture des bâtiments et l'occupation des locaux) avec leur passé et ainsi proposer une typologie des différentes formes d'intervention urbaine subies par ces sites et de leurs conséquences sur la représentation possible des effets néfastes qu'ils ont pu générer.

\section{Créer un nouveau paysage}

22 Une première catégorie comprend les espaces ayant accueilli une ou plusieurs industrie(s) mais dont les marques physiques originales ont disparu, qu'ils soient en friche ou bien qu'après l'obligation de dépollution des sols (Article L 556-1 du Code de l'environnement complété par la Loi n²014-366 du 24 mars 2014 dite Alur), ils se voient attribuer une nouvelle fonction, voire identité, rendant difficile la représentation de son passé.

\section{Détruire pour « retourner à la nature »}

Notre liste répertorie huit sites reconvertis en espace vert ${ }^{11}$. Les anciennes carrières de gypse et leurs unités annexes (plâtrières, briqueteries, maçonneries) restent les sites les plus souvent concernés à l'exception d'une cristallerie au Bourget et de l'ancienne usine Kodak à Sevran ${ }^{12}$.

D'un espace hébergeant une activité source de nuisances et de pollutions (des sols, de l'air, de l'eau) émerge alors un espace vert, dépollué (ou ayant l'apparence de l'être), reflet des préoccupations actuelles de la ville "durable ", qu'elles soient paysagères, sociétales et/ou environnementales. La reconversion de carrières en parcs et jardins demeure relativement traditionnelle. Les cavités et galeries souterraines créées lors de l'exploitation des carrières fragilisent la stabilité du sol et ce même si un comblement est effectué. Le remblayage et "le retour à la nature" de ces espaces sont alors privilégiés, actions répondant aux enjeux de développement durable (Dumesnil et Ouellet, 2002), de contrôle de l'étalement urbain (Veschambre, 2005) ou de la réintroduction de la biodiversité en ville. Par exemple, à Bagnolet, le parc départemental Jean-Moulin les Guilands a émergé en lieu et place d'anciennes carrières de gypse (illustration 3). 


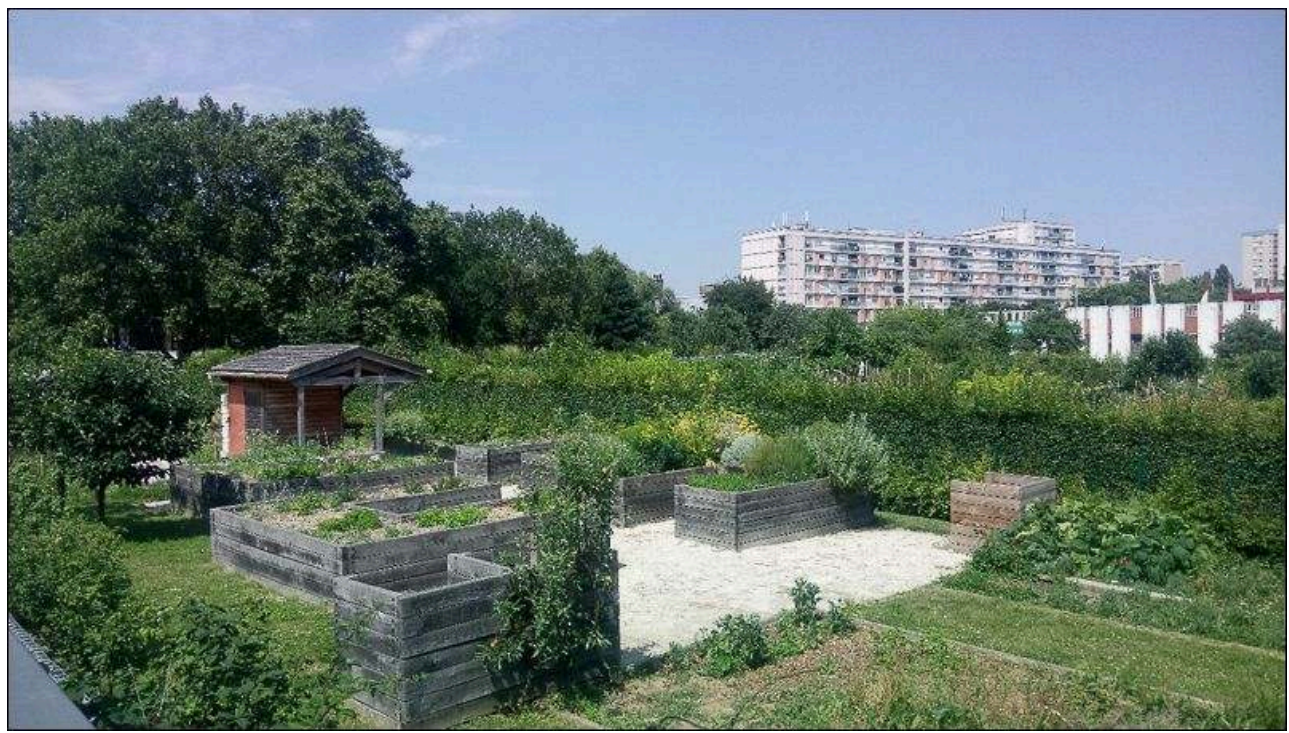

Auteur : A. Croisé, juillet 2018

La transformation spectaculaire du paysage (y compris de sa topographie) et sa métamorphose en espace supposément sain (Boutefeu, 2007) compromettent le lien du site avec son passé pollué. Pourtant, l'exploitation du gypse génère des poussières provoquant des pathologies pulmonaires graves chez ses travailleurs telles que les pneumoconioses. L'analyse textuelle montre que la description de l'exploitation du gypse reste généralement sommaire et factuelle (date d'installation, de fermeture du site et/ou description de l'activité et/ou surface de la carrière, et/ou le devenir du site) et n'évoque que de manière positive l'activité ou les changements opérés. Une exception est constituée par les anciennes carrières de Gagny qui sont qualifiées de friche « industrielle dangereuse ». Néanmoins, ce danger n'est alors pas explicité et le caractère potentiellement néfaste de cette friche est immédiatement contrebalancé par l'affirmation de sa reconversion en ces termes: "Après un diagnostic et une concertation auprès des Gabiniens, Michel Teulet, Maire de Gagny, élabore un projet [celui de] faire émerger de cette friche industrielle dangereuse un espace de vie de 12 hectares dédié à la biodiversité ". Le contraste entre un " avant » dangereux et un après sain est encore plus frappant dans le cas de la friche Kodak (traitement des films et de photographies). Après avoir pollué les sols (aux hydrocarbures, aux PCB-PCT et aux solvants halogénés) ${ }^{13}$, la nappe souterraine (aux HAP et aux solvants halogénés) ${ }^{14}$ et contribué au développement de cancers d'origine professionnelle ${ }^{15} \mathrm{l}^{\prime}$ usine est détruite et son terrain dépollué. En friche, le site reste accessible avant d'être finalement sélectionné dans le cadre du programme Nature 2050 pour devenir un "îlot de biodiversité ». La toponymie utilisée alentour participe elle-même de ce basculement puisqu'elle évoque majoritairement la nature, en particuliers autour des parcs (sentiers « des buttes» et des « ravins »). Plus le temps passe et les mutations du territoire sont profondes, plus la distance augmente entre les populations habitantes et ce passé industriel. Sont-elles conscientes que l'ouverture d'un espace vert sur une ancienne friche est souvent l'alternative imaginée quand les locaux ne sont pas réutilisables ou quand les terrains sont trop pollués ? En effet, cet aménagement ne nécessite pas de lourds travaux de dépollution, contrairement à la construction de logements ou d'équipements publics. 
Le foncier étant tout de même recherché en Seine-Saint-Denis, les friches industrielles deviennent généralement des terrains attractifs d'un point de vue économique et social (De Roo, 1987; ADEME, 2014) et ce malgré la lourdeur des travaux de dépollution à engager. La construction d'infrastructures nouvelles révèle les mêmes mécanismes d'invisibilisation des effets pathogènes des anciennes industries bien que le passé du site, semble ici plus facilement convoqué afin d'évoquer les transformations positives des espaces concernés.

\section{Détruire pour reconstruire}

D'après notre travail de terrain, 52 industries (de notre liste de sites) ont vu leurs locaux détruits libérant un espace pour diverses infrastructures aux activités mixtes: commerciales (du café ou de l'hôtel aux grandes surfaces), résidentielles (de la maison à l'immeuble), industrielles et/ou de services (de bureaux). Elles répondent plus ou moins, aux besoins actuels de la population départementale ou francilienne. Ainsi, en lieu et place de l'usine Japy (machines à écrire) à Saint-Ouen se trouvent une résidence universitaire et des logements; de même les gazomètres et entrepôts de la société du Gaz de France à Saint-Denis ont été remplacés par des espaces verts, des logements, des bureaux et des sièges d'entreprises (illustration 4) tandis que l'usine Olida (de boites de conserves) a été remplacée par un cinéma à Epinay-sur-Seine. Comme pour les espaces verts, les paysages et les fonctions changent. La toponymie reste bien souvent le seul moyen, sur place, de faire référence au passé (le parc, le Temps des Cerises fait référence à une chanson ouvrière, illustration 4). Et si celui-ci est plus largement évoqué sur les sites web des mairies, son évocation demeure factuelle (date d'installation, surface, évolution de l'activité, devenir du site, etc.) et les aspects nocifs des activités sur leur environnement ne sont que peu, voire pas, évoqués, la santé des travailleurs n'étant, quant à elle, pas du tout mentionnée. Néanmoins, constatons que plus de la moitié des sites sur lesquels les communes communiquent concernent des unités de production qui ont totalement disparu. La construction de bâtiments neufs contribue à la négation paysagère du passé industriel (Edelblutte, 2011) mais n'engendre pas nécessairement son oubli par les institutions ou par l'histoire. D'autant plus que la vente d'un terrain ou la construction d'une nouvelle infrastructure sur une parcelle nécessite bien souvent de savoir à qui et à quoi était destiné le terrain. 
Illustration 4 - Le parc du Temps des Cerises entouré des locaux de la SNCF et de SFR en lieu et place des anciens gazomètres de la société du Gaz de France à Saint-Denis

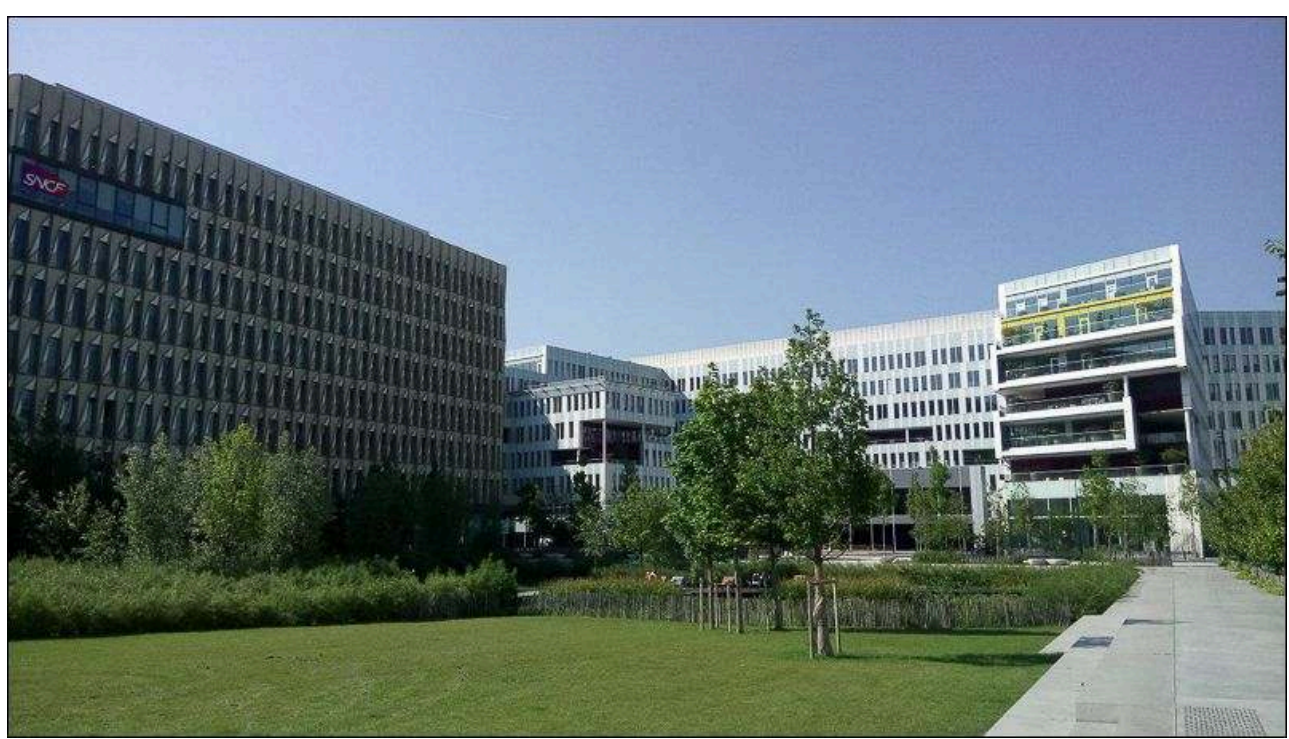

Auteur : A. Croisé, mai 2018 de la dépollution, ceci comme pour mieux tourner la page de la période d'exposition. Ainsi, l'entreprise de freins ferroviaires Westinghouse à Sevran avait pollué ses sols (aux hydrocarbures, solvants halogénés et TCE), sa nappe phréatique (arsenic, BTEX, HAP, sélénium, solvants halogénés et TCE) et exposé ses travailleurs à de nombreux cancérogènes (acides minéraux, amiante, benzène, chrome, fumées de soudage, HAP, mines de fer, nickel, plomb, silice, solvants chlorés et huiles de coupe ${ }^{16}$ ) pendant plus de 80 ans. L'arrêt des activités dans les années 1990 conduit à la destruction des bâtiments et à la dépollution des sols et des eaux souterraines au début des années 2010. Le site web municipal l'évoque à travers les questions de responsabilité, de niveau et de modalités de dépollution. Cependant, ce rappel est occulté par le projet de $60000 \mathrm{~m}^{2} \mathrm{de}$ logements et de services. La nouvelle toponymie, le quartier s'appelant désormais "Westing'Plaza », fait également référence au passé industriel mais en utilisant la notoriété et la puissance de la multinationale en la combinant à l'évocation de l'hôtel de luxe new-yorkais (« le Plaza »).

passé industriel n'est donc pas occulté ni dans les discours présent sur le web ni par la toponymie. Cependant, son évocation rappelle davantage la puissance industrielle que la condition des travailleurs.

\section{Conserver une infrastructure mobilisable}

Si, par principe, les bâtiments sont censés porter dans leur architecture, leur configuration et leur aspect général, la trace physique de l'activité qui les a occupés (Jeudy, 2008), leur maintien dans le paysage pourrait contribuer à entretenir sa mémoire sur le temps long (Sgard, 2007). Néanmoins, leur réhabilitation, les choix de mise en valeur architecturale et la communication qui en est faite aboutissent à une mémoire très partiale. Ainsi, Antoine Furio $(2012$, p.45) note : «à notre connaissance, 
sur toutes les reconversions réalisées en Seine-Saint-Denis en 35 ans, aucune ne restitue de manière satisfaisante le sens du lieu ».

\section{Conserver un bâtiment à l'architecture originale...}

31 Ce qui fait patrimoine [6 ici incarné par le bâtiment même et son architecture originale et/ou (a)typique (typique pour l'époque, atypique de nos jours). À l'origine, ces constructions incarnent et symbolisent la puissance de l'industriel. Conserver cette architecture prolonge cette symbolique et, si elle glorifie une activité, un savoir-faire technique, elle occulte en contrepartie les effets néfastes de l'activité sur la santé des ouvriers et des riverains.

La plupart des bâtiments conservés (à l'architecture originale) sont ré-exploités et réinvestis par des activités culturelles (la fabrique d'allumette à Aubervilliers accueille la documentation française puis divers services municipaux et à destination des entreprises et enfin, plus récemment, l'Institut National du Patrimoine), économiques ou résidentielles (l'entreprise de bicycles, tricycles et quadricycles Gladiator au PréSaint-Gervais a été transformée en logements et en commerces au rez-de-chaussée) conduisant à réattribuer à la fois une fonction et de nouvelles représentations aux sites (Luxembourg, 2013). Ces opérations engagent à la fois le propriétaire du site et la mairie, car le projet doit correspondre au Plan Local d'Urbanisme (PLU). L'architecture industrielle est alors un argument mobilisé pour justifier puis valoriser l'opération d'urbanisme et ne peut être en ce sens un rappel des nuisances liées à l'activité passée. La visite du patrimoine industriel que le Département organise à Montreuil n'évoque que les anciennes industries du luxe dont une partie des bâtiments conservés sont relativement imposants (par exemple les entrepôts de traitement de peaux de lapin Hugon, illustration 5 et la Société parisienne de tranchage et de déroulage, illustration 6) et dont les activités sont renommées (distillerie, jouets, porcelaine, etc.) voire primées. Le Département ne véhicule ainsi qu'une image très positive de son passé industriel alors que ces activités ont nécessité l'emploi de produits ou matériaux (peinture, teinture, bois, solvants..) et de procédés (distillation, trempage, teinture, séchage des peaux, etc.) néfastes pour la santé. 
Illustration 5 - Les anciens locaux d'Hugon Frères à Montreuil

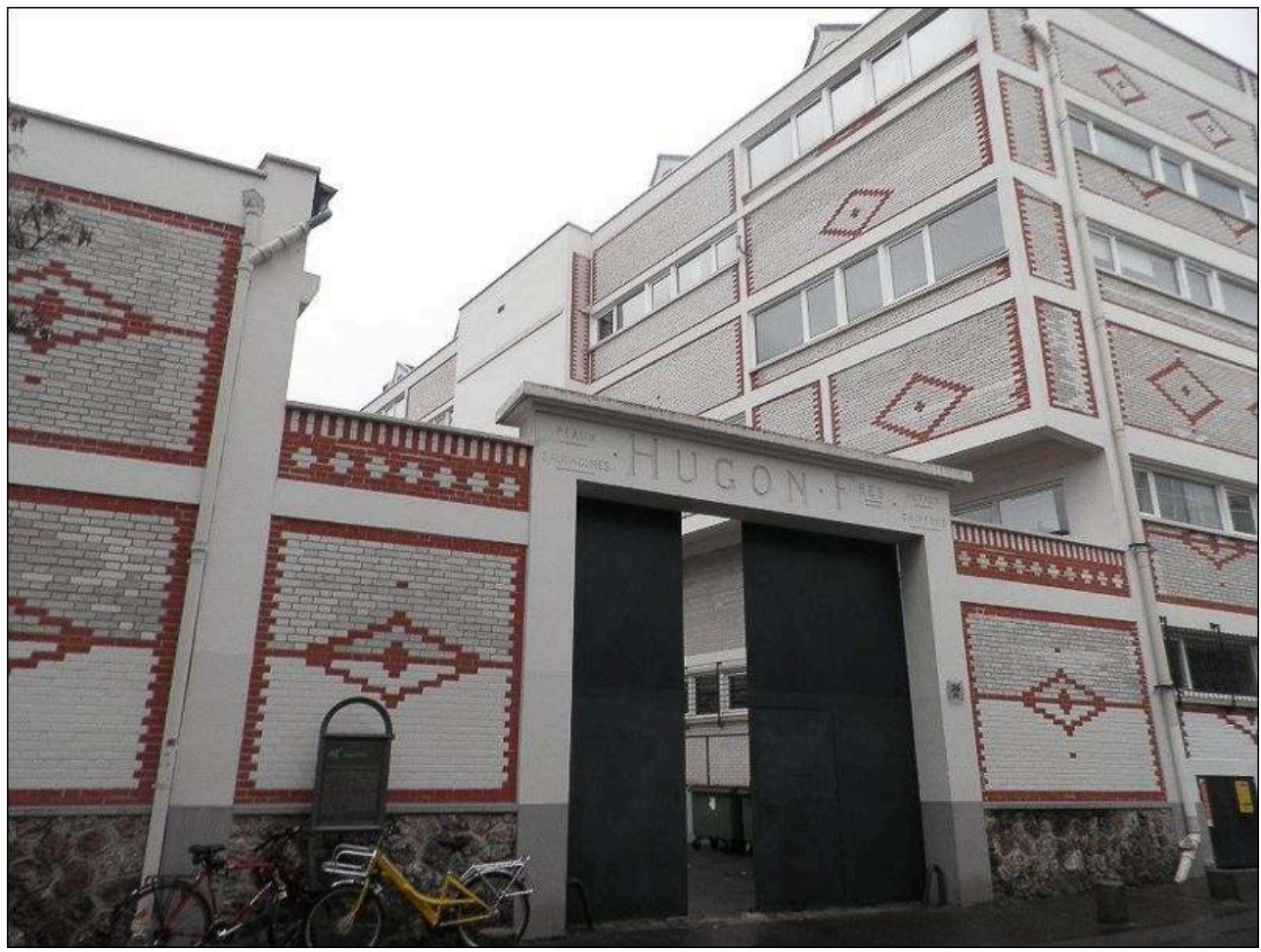

Auteur : A. Croisé, février 2018.

Illustration 6 - Les locaux de l'ancienne Société Parisienne de Tranchage et de Déroulage (SPTD) à Montreuil

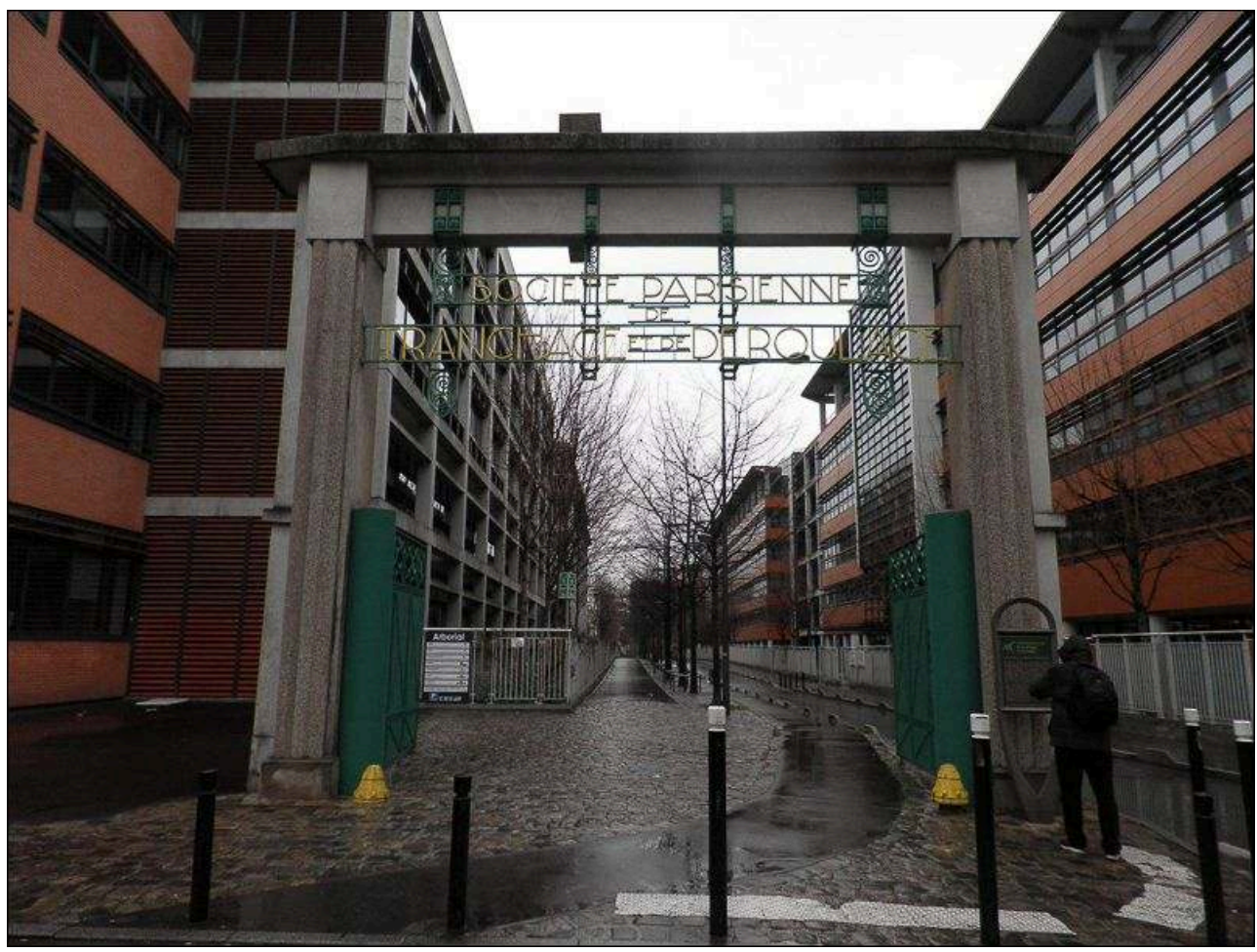

Auteur : A. Croisé, février 2018. 


\section{Dissimulé tel un objet d'art}

Les traces peuvent être beaucoup plus difficiles à percevoir. Toujours à Montreuil, l'ancien château d'eau de la distillerie Pernod (87 rue de Paris) (illustration 7) et trois fours de la porcelainerie Samson (14 rue de la Révolution) sont dissimulés à l'intérieur de cours d'immeubles. L'ensemble des bâtiments, à l'exception de ces infrastructures, ont été détruits suite à l'arrêt des activités et à la volonté de la commune de réinvestir l'espace, réduisant considérablement la visibilité (physique) du passé industriel de la commune tout en conservant des éléments de ses industries du luxe. Parmi l'ensemble des bâtiments, n'est alors conservé que ce qui a valeur à l'être selon la société d'économie mixte pour l'usine Pernod (le château d'eau pour sa valeur architecturale) et la commission des monuments historiques pour la porcelainerie Samson (les fours ont été classés). Ces infrastructures, fortes de la valeur qui leur a été accordée, se trouvent au cœur d'une restructuration urbaine, elles sont alors une exception au milieu d'un nouvel espace qui les dissimule (derrière des bâtiments) mais qui a été pensé autour d'elles et les intègre. En effet, le château d'eau se voit difficilement de l'extérieur tandis que les fours de la porcelainerie Samson se trouvent derrière un grillage dans une cour fermée par un code d'accès. Un panneau d'information de la ville de Montreuil, dans la rue adjacente, signale la présence de l'ancienne porcelainerie ${ }^{17}$ tout comme le site web de la commune. La «dissimulation» de ces infrastructures uniques nous conduit à les considérer comme des joyaux (puisque exceptionnels) cachés, que seuls les riverains ou les personnes s'intéressant au passé industriel ${ }^{18}$ peuvent contempler. Ce n'est plus tant l'activité qui focalise l'attention (et donc sa possible nocivité), mais davantage l'idée et l'envie de découvrir une infrastructure unique et exceptionnelle. 
Illustration 7 - L'ancien château d'eau de l'usine Pernod, entouré d'immeubles résidentiels et de bureaux à Montreuil

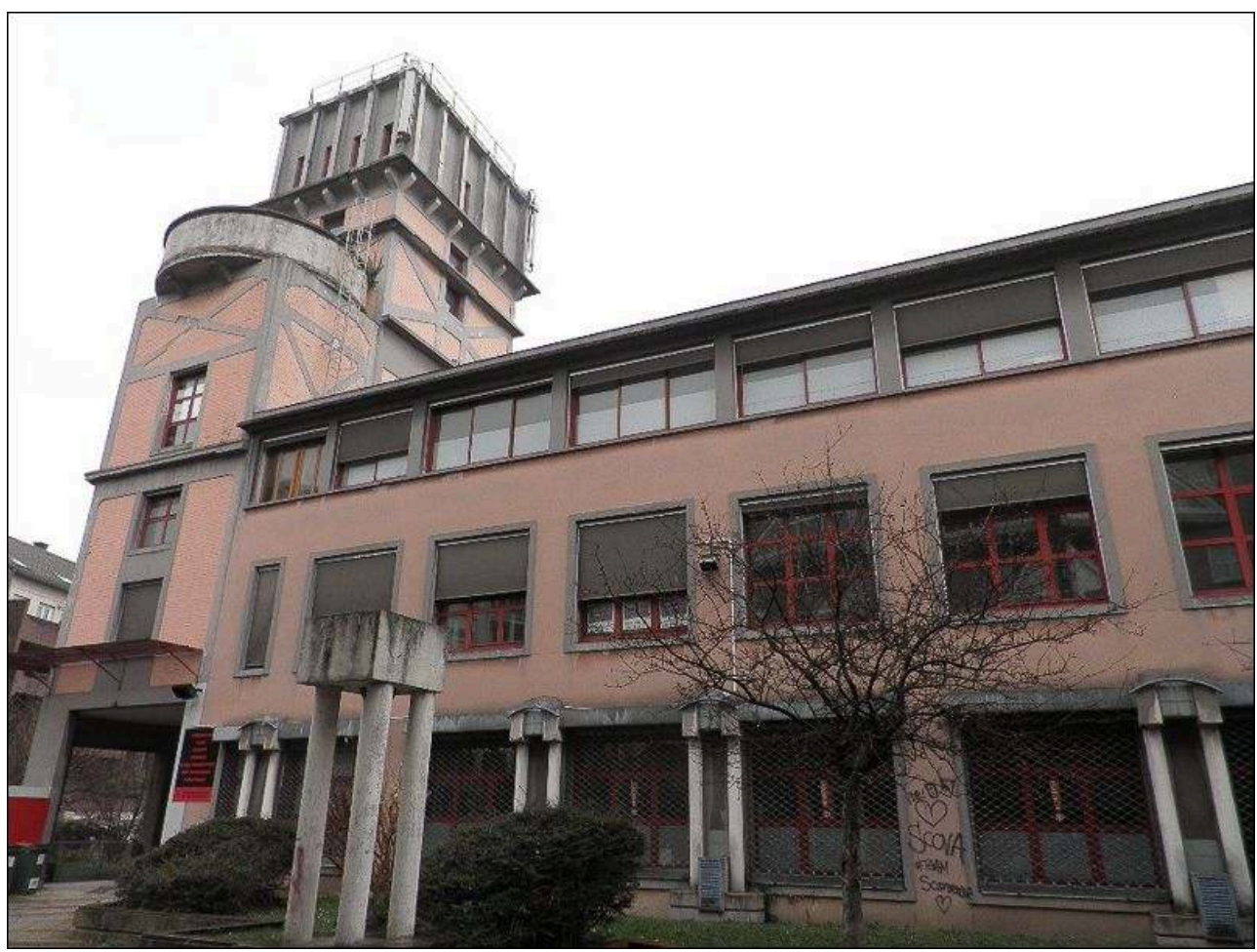

Auteur : A. Croisé, février 2018. comme au figuré. En effet, même dans le cas du maintien et de la réhabilitation d'une usine, les cheminées sont démolies, principalement pour des questions de sécurité. Elles portent une représentation ambivalente évoquant la puissance économique mais aussi le rejet des fumées (Massard-Guilbaud, 2010). Quand l'une d'elles est conservée, elle devient une œuvre d'art du fait de sa rareté, contribuant à une esthétisation du paysage au point d'en connaître des avatars: la cheminée disparue de la société de tranchage à Montreuil a ainsi laissé place à une œuvre de même taille la représentant.

Ce focus sur l'architecture, la beauté et l'exceptionnalité des infrastructures et la renommée des activités nous confronte aux questions que pose la transmission de l'histoire industrielle. Ces opérations de conservation/réhabilitation font écho aux inventaires du patrimoine industriel qui se concentrent sur les aspects architecturaux. Cependant, en confrontant ces inventaires et les sites effectivement présents sur les sites web des communes, nous constatons que seuls $30 \%$ d'entre eux se retrouvent dans les inventaires du patrimoine.

\section{Conserver ceux qui se fondent dans le paysage actuel}

Certains de ces bâtiments ne possèdent pas de d'éléments architecturaux notables rappelant l'ère industrielle (toits en sheds, briques rouges, cheminées, grandes ouvertures, etc.) (Real, 2015). Nous en avons recensé trois, tous inclus dans les parcours $\mathrm{du}$ «patrimoine industriel» des communes. Ceux de Montreuil sont inclus dans celui organisé par le Département et s'accompagnent exceptionnellement de plaques informatives municipales témoignant de la volonté de la mairie de communiquer sur 
son passé industriel. Les deux sites concernés sont ceux de l'ancienne entreprise Jumeau et des studios Pathé (illustrations 8 et 9) dont la structure, quasiment conservée à l'identique, est dissimulée derrière un mur et ne se distingue pas d'une immeuble de logement ou de bureaux. Les locaux de la savonnerie des deux mondes à Epinay-surSeine s'intègrent également parfaitement au paysage résidentiel de la rue (illustration 10).

Illustration 8 - Les anciens locaux de l'entreprise de poupées Jumeau à Montreuil

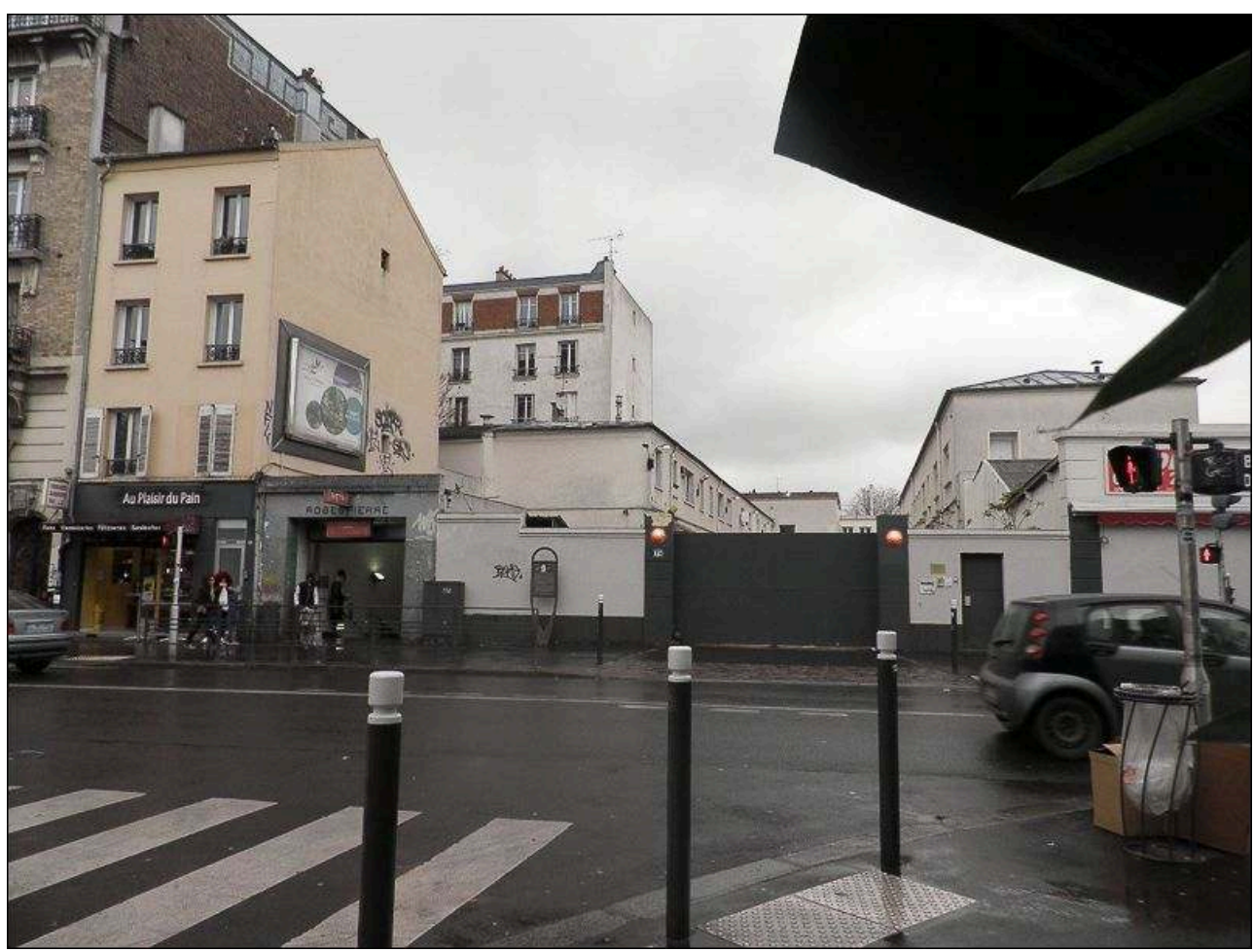

Auteur : A. Croisé, février 2018. 
Illustration 9 - Les anciens studios Pathé à Montreuil

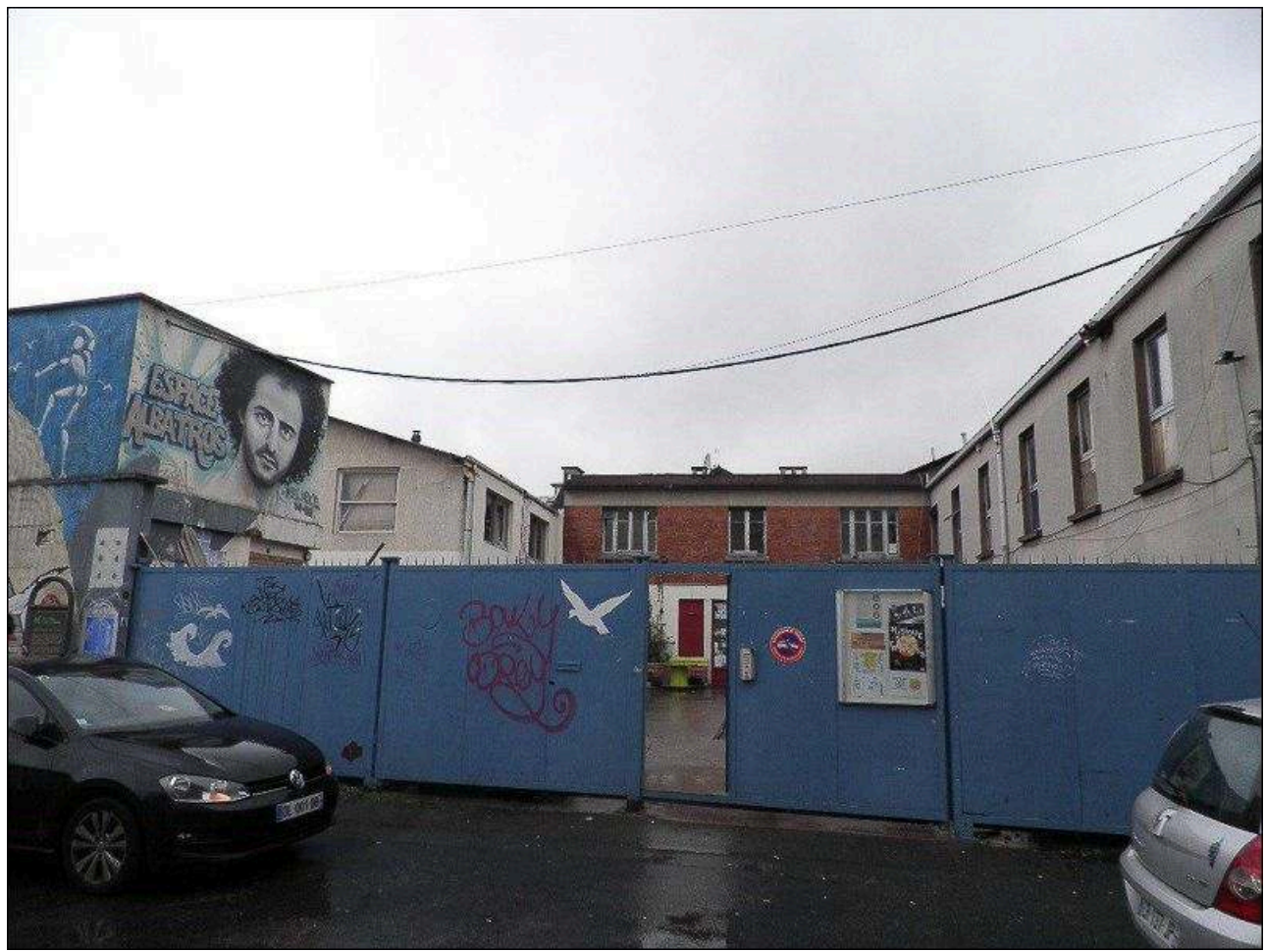

Auteur : A. Croisé, février 2018.

Illustration 10 - Les locaux de la savonnerie des deux Monde à Epinay-sur-Seine dissimulés derrière le portail vert

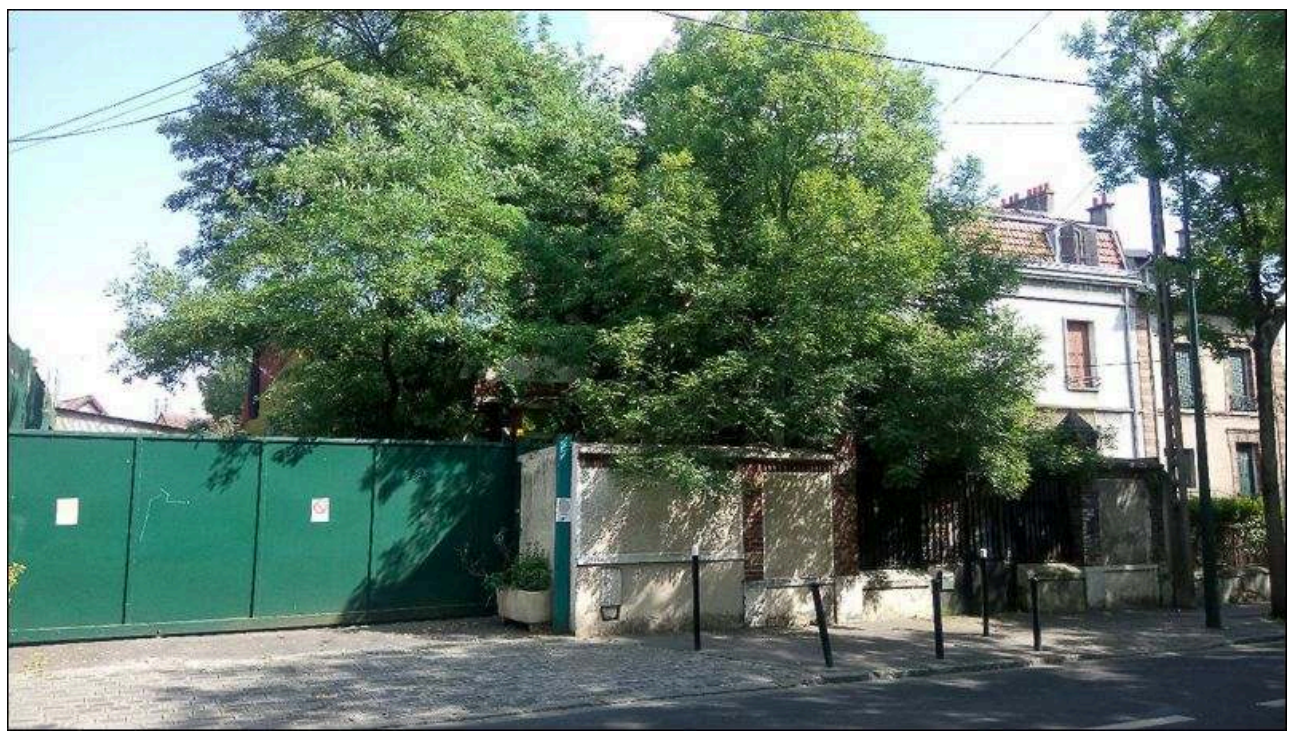

Auteur: A. Croisé, juin 2018

Cette insertion dans le paysage urbain ne permet pas au néophyte de faire spontanément un lien entre une activité industrielle et ces bâtiments. Le panneau (pour les studios Pathé) ne relaie que des informations brèves, factuelles tout comme les sites web des communes. Contrairement aux réhabilitations vues précédemment, 
l'architecture importe peu et me bâtiment acquiert un statut de patrimoine ordinaire de la Seine-Saint-Denis puisqu'il s'intègre parfaitement au paysage.

\section{Conclusion}

38 Bien que les bases de données disponibles aient leurs limites, la combinaison de plusieurs d'entre elles, associées aux savoirs engendrés sur les conditions de travail des ouvriers et sur les pollutions environnementales des industries (Massard-Guilbaud, 2010 ; Guillerme et al., 2004) permettraient d'établir des liens, quoique potentiels, entre un site, son activité et ses effets sur la santé tant des travailleurs que des riverains. Tel n'est pas le cas et la mise en patrimoine de l'héritage industriel n'y contribue pas.

39 Malgré l'existence d'un passé industriel, socialement, économiquement et politiquement structurant en Seine-Saint-Denis, la mémoire institutionnelle et ouvrière semble très lacunaire quand des pathologies se déclarent, et ce, malgré la médiatisation $\mathrm{du}$ produit ou process mis en cause, comme peut l'être l'amiante. Cancérogène surmédiatisé en France des suites du « scandale de l'amiante » (Henry, 2007), facteur de risque reconnu de cancers, l'amiante a été utilisée dans l'industrie locale. Pourtant, le lien entre l'existence de patients atteint de cancers broncho-pulmonaires et ces activités industrielles n'est pas faite. Bien que les municipalités du département soient attachées à maintenir une mémoire ouvrière et industrielle du territoire, les conséquences sanitaires des activités industrielles ne transparaissent pas dans la présentation du patrimoine que ce soit dans les sites web ou dans les visites.

40 Cela tient à plusieurs facteurs, certains épidémiologiques, certains urbanistiques. La pathogénicité de l'usine du Comptoir des Minéraux et Matières Premières (CMMP) à Aulnay-Sous-Bois ne révèle l'étendue de ses conséquences sanitaires qu'après sa fermeture. Il a fallu une mobilisation citoyenne et scientifique (à l'origine de plusieurs rapports) et une communication importante (via divers médias) pour identifier de potentielles victimes. Malgré cette médiatisation, le site web de la municipalité n'évoque pas l'aspect pathogène de son ancienne usine alors qu'elle pourrait relayer les messages diffusés par le collectif des victimes et riverains du CMMP. Sur le terrain, seule une dalle en béton armé marque l'emplacement de l'ancienne usine alors que le collectif demande à la mairie l'installation d'une stèle commémorative en hommage aux victimes. Malgré l'impact sanitaire, la souffrance générée par l'usine n'a pas sa place dans le paysage.

41 Le traitement des anciens sites industriel, et en particulier leur institution comme objets d'un patrimoine « digne » d'être transmis, réduit, de fait, leur apparente nocivité tandis que les nouveaux sites ont tendance à être relégués dans des zones industrielles ou d'activité limitant la proximité physique avec les habitants. De plus, la valorisation du passé doit être mise en regard des représentations dominantes du département: une banlieue rongée par le chômage et la pauvreté visibles aux yeux de tous notamment depuis les émeutes de 2005 (Avenel, 2009). Indiquer les risques sanitaires est contradictoire avec l'objectif de valoriser l'image de la Seine-Saint Denis. Par ailleurs, la population et ses représentants se battent dans le présent contre des inégalités sociales et économiques qui ont un impact immédiat sur le quotidien des Séquano-dionysiens, relayant en arrière-plan des considérations sanitaires, et ce d'autant plus quand l'emploi « ouvrier » s'inscrit dans la précarité. En effet, comment dénoncer la pathogénicité du lieu qui fait vivre celui qu'il rendra peut-être, un jour, 
malade ? Une forme d'omerta se crée, et la combinaison des divers facteurs conduit à recréer pour les nouveaux habitants, une mémoire de l'industrie séquano-dionysienne fantasmée et romantique.

\section{BIBLIOGRAPHIE}

Agence de l'environnement et de la maîtrise de l'énergie (ADEME), 2014. La reconversion des sites et des friches urbaines pollués. Publication de l'ADEME, $12 \mathrm{p}$.

Ambrosino C., Andres L., 2008. Friches en ville : du temps de veille aux politiques de l'espace. Espaces et sociétés, vol. 134, n 3, p. 37-51.

Avenel C., 2009. La construction du « problème des banlieues » entre ségrégation et stigmatisation. Journal français de psychiatrie, $\mathrm{n}^{\circ} 3$, vol. 34, p. 36-44.

Boutefeu E., 2007. La nature en ville : des enjeux paysagers et sociétaux. Géoconfluences [En ligne]. http://geoconfluences.ens-lyon.fr/doc/transv/paysage/PaysageViv.htm

Calvez M., 2009. Les signalements profanes de clusters de cancers : épidémiologie populaire et expertise en santé environnementale. Sciences sociales et santé, vol. 27, n² 2, p. 79-106.

Dalbavie J., 2012, Entre patrimoine et mémoire collective, la tombe de Georges Brassens. Questions de communication, $\mathrm{n}^{\circ} 22$, p. 103-122.

Davallon J., 2002. Tradition, mémoire, patrimoine. In Schiele B. (dir.), Patrimoines et Identités. Québec. Musée de la Civilisation-Éd. MultiMondes, p. 42-64.

Del Biondo L., Edelblutte S., 2016. Le paysage des anciennes villes-usines européennes : un nouveau patrimoine entre négation, alibi, reconnaissance et complexité des jeux d'acteurs. Annales de Géographie, $\mathrm{n}^{\circ} 711$, p. 466-489.

De Roo P., 1987. Les friches industrielles en Île-de-France. Mappemonde [En ligne], 87/1, p. 11-13. https://www.mgm.fr/PUB/Mappemonde/M187/p11-13.pdf

Di Méo G., 2007. Processus de patrimonialisation et construction des territoires. Actes du colloque Patrimoine et industrie en Poitou-Charentes. Connaître pour valoriser. Poitiers - Châtellerault, Geste, $\mathrm{p}$. 87-109.

Direction régionale des entreprises, de la concurrence, de la consommation, du travail et de l'emploi (DIRECCTE) d'île-de-France, 2011. Évolution de l'emploi salarié en Seine-Saint-Denis, 52 p.

Djament-Tran G., 2014. Patrimonialisation et territorialisation dans la nouvelle étape du capitalisme: le cas de Plaine Commune. $2^{e}$ colloque international du CIST Fronts et frontières des sciences du territoire. Paris, p. 166-172.

Dumesnil F., Ouellet C., 2002. La réhabilitation des friches industrielles: un pas vers la ville viable? Vertigo, vol. 3, n², p. 1-19.

Edelblutte S., 2008. Paysages et territoires du patrimoine industriel au Royaume-Uni. Revue Géographique de l'Est, vol. 48, n 1-2, p. 1-29. 
Edelblutte S., 2010. Paysages et territoires de l'industrie en Europe : héritages et renouveaux. Paris, Ellipses, $272 \mathrm{p}$.

Edelblutte S., 2011. Que reste-t-il de l'industrie après la désindustrialisation ? De la négation au patrimoine industriel. Bulletin de l'Association de géographes français, vol. 88, n 2, p. 150-163.

Fourcaut A., 2007. Les banlieues populaires ont aussi une histoire. Revue Projet, vol. 299, n 4 , p. 7-15.

Furio A., 2012. Trente ans de reconversion en Seine-Saint-Denis. L'Archéologie industrielle en France, $\mathrm{n}^{\circ} 60, \mathrm{p} .40-47$.

Gollac M., Volkoff S., 2007. Les conditions de travail. Paris, La Découverte, 128 p.

Guillerme A., Lefort C., Jigaudon G., 2004. Dangereux, insalubres et incommodes: paysages industriels en banlieue parisienne, XIX ${ }^{e}-X^{e}$ siècles. Seyssel, Champ Vallon, $343 \mathrm{p}$.

Henry E., 2007. Amiante : un scandale improbable. Res publica, Presses universitaires de Rennes, $312 \mathrm{p}$.

Hill A. B., 1965. The Environment and Disease: Association or Causation? Proceedings of the Royal Society of Medicine, vol. 58, $\mathrm{n}^{\circ}$ 5, p. 295-300.

Jeudy H.-P., 2008. Temporalités citadines : la reconquête de l'espace public. In Cousin S., Da Lage E., Debruyne F., Vandiedonck D. (dir.), Le sens de l'usine. Paris, Créaphis, p. 69-74.

Lebeau B., 2014. Une « banlieue créative » dans le Grand Paris ? EchoGéo [En ligne], Sur le Vif. http://journals.openedition.org/echogeo/15202

Letté M., 2011. L'histoire des débordements industriels à l'origine de conflits autour de l'environnement. Annales des Mines - Responsabilité et environnement, vol. 62, n² 2, p. 43-50.

Lévy J., Lussault M. (dir.), 2013. Dictionnaire de la géographie et de l'espace des sociétés. Paris, Belin, $1127 \mathrm{p}$.

Luxembourg C., 2013. Patrimonialiser, revitaliser, habiter l'industrie en ville : une question politique et sociale vivante plus qu'une simple question de renouveau urbain. Revue Géographique de l'Est, vol. 53, n 3-4, p. 1-17.

Lysaniuk B., Croisé A., Tabeaud M., Counil E., 2015. La difficile compilation d'information(s) géographique(s) relative(s) à un cancérogène : étude pilote sur les expositions professionnelles à l'amiante en Seine-Saint-Denis. Pollution atmosphérique, n² 225, p. 1-16.

Massard-Guilbaud G., 2010. Histoire de la pollution industrielle. France, 1789-1914. Paris, Éditions de l'EHESS, $404 \mathrm{p}$.

Nora P., 1984. Les lieux de mémoire, tome 1 : la République. Paris, Gallimard, 720 p.

Osadtchy C., 2015. Conflits environnementaux en territoire industriel. Réappropriation territoriale et émergence d'une justice environnementale : le cas de l'étang de Berre et de Fos-sur-Mer. Thèse de géographie sociale et régionale, Université du Maine, 492 p.

Real R., 2015. Reconversions. L'architecture industrielle réinventée. In Situ, n² 26, p. 1-72.

Redon F., 2018. « Sites et sols pollués : héritage du passé industriel en Seine-Saint-Denis ». Liaison, $\mathrm{n}^{\circ} 179$.

Retaillé D., 2011. Introduction à une géographie des conflits. L'Information géographique, vol. 75, $\mathrm{n}^{\circ} 3$, p. 6-22.

Ricœur P., 2000. La mémoire, l'histoire, l'oubli. Paris, Éditions du Seuil, 675 p. 
Sierra A., 2000. Gestion et enjeux des espaces urbains à risque d'origine naturelle - Les versants et les quebradas de Quito, Equateur. Thèse de doctorat, Université Paris VIII, Centre de Recherche en Analyses Géopolitiques, 326 p.

Sgard A., 2007. Mémoires, lieux et territoires. Presses universitaires de Rennes, p. 105-117.

Siméon O., 2014. Quel patrimoine industriel pour quelle vision de l'histoire ? Le cas de la GrandeBretagne. L'Homme et la société, vol. 192, n², p. 15-30.

Tuaillon-Demésy A., 2013. Mémoire, histoire et patrimoine. Une illustration : la pratique de l'histoire vivante médiévale. Émulations, $\mathrm{n}^{\circ} 11$, p. 2-8.

Veschambre V., 2005. Le recyclage urbain, entre démolition et patrimonialisation : enjeux d'appropriation symbolique de l'espace. Norois, n) 195, p. 79-92.

Vieillard-Baron H., 2011. La Plaine Saint-Denis : un ancien territoire industriel au centre des contradictions métropolitaines. Bulletin de l'Association de géographes français, vol. 88, $\mathrm{n}^{\circ} 2$, p. 164-173).

\section{NOTES}

1. La Base de données des sites et sols pollués (BASOL, disponible sur: https:// basol.developpement-durable.gouv.fr/recherche.php) recense, le 17 mai 2018, 63 sols pollués notamment au chrome, au plomb, au mercure, au cyanure, aux hydrocarbures, aux solvants, etc.

2. Il s'agit d'un cancer de la plèvre. L'exposition à l'amiante est - à ce jour - le seul facteur de risque connu, associé à cette pathologie.

3. Le terme pathogène fait référence à « tout processus qui engendre des troubles ", qui « induit une pathologie » (Dictionnaire de l'Académie Nationale de Médecine).

4. «La réhabilitation [est une] opération de rétablissement d'un édifice ou d'un ensemble d'immeubles [...] dans ses capacités à abriter des activités et des habitants » contrairement à la rénovation qui "fait table rase pour édifier selon les normes en vigueur » (Lévy et Lussault, 2013). La reconversion urbaine est « une action volontaire de requalification spatiale, sociale, économique et culturelle d'espaces industriels en crise » (ibid.)

5. Les chiffres sont arrondis. Le taux d'évolution est calculé à partir des données disponibles sur le site de l'INSEE : Catégorie socioprofessionnelle des actifs - Données harmonisées RP1968-1999 et $\mathrm{du}$ dossier complet départementale (https://www.insee.fr/fr/statistiques/2011101? geo=DEP-93, consulté le 01/11/2018).

6. «Le constat et la prise de conscience du sursis vécu par les dernières usines face aux mutations des activités et aux transformations du paysage. C'est pourquoi s'est imposée l'urgence de mener une opération sur la commune avant qu'il ne soit tard» (inventaire du patrimoine industriel de Saint-Ouen). «La désindustrialisation qui a frappé l'ensemble de ces communes a laissé sur le terrain peu de traces visibles de cette activité» (inventaire du patrimoine industriel de Montreuil).

7. Le préambule de l'inventaire du patrimoine de Romainville insiste sur l'importance de « constituer une " mise en mémoire» des sites. Les bâtiments seraient considérés comme "les témoins vivants de l'activité ancienne ». Des propos identiques se retrouvent dans le préambule de l'inventaire de Pantin.

8. La loi $n^{\circ} 85-98$ du 25 janvier 1985 a été abrogée au début des années 2000. Elle prévoyait la sollicitation des archivistes en cas de péril ou de liquidation d'une société. Elle n'a pratiquement jamais été appliquée. La loi L642-23 du code du commerce prévoit «avant toute vente ou toute destruction des archives du débiteur, le liquidateur en informe l'autorité administrative 
compétente pour la conservation des archives. Cette autorité dispose d'un droit de préemption. La destination des archives du débiteur soumis au secret professionnel est déterminée par le liquidateur en accord avec l'ordre professionnel ou l'autorité compétente dont il relève. » Elle ne serait pas non plus appliquée.

9. Pour les communes de Saint-Ouen et Epinay-sur-Seine un parcours historique est proposé sur leur site web avec des informations concernant les sites visités, pour Montreuil, un guide travaillant pour le département nous propose la découverte du patrimoine industriel lors d'une visite de $2 \mathrm{~h}$.

10. Pour effectuer cette recherche, nous avons consulté l'ensemble des onglets présents sur les sites web et poursuivi par une recherche via plusieurs mots-clés comme "industrie ", " usine ", « carrière ", etc.

11. Le parc de la Garenne à Villemomble, le parc Decesari à Rosny-sous-Bois, le parc des Beaumonts à Montreuil, le parc départemental Jean-Moulin les Guilands à Bagnolet, le parc forestier du Bois de l'Étoile à Gagny, le parc de Sévigné à Livry-Gargan, le square Charles de Gaulle au Bourget et la friche Kodak à Sevran.

12. Le parc de la Poudrerie de Sevran n'est pas inclus dans cette catégorie puisque celui-ci existait au temps de l'activité de la Poudrerie. L'activité de la Poudrerie nécessitait l'utilisation de poudres et de produits chimiques pour la préparation d'explosifs. Ces matériaux ont largement contaminé les sous-sols du parc qui commencent tout juste à être dépollués (janvier 2018).

13. Les $\mathrm{PCB}$ (polychlorobiphényles) et PCT (polychloroterphényles) sont considérés comme les polluants organiques persistants. Les PCB sont classés par le Centre International de Recherche sur le Cancer (CIRC) comme cancérogènes certains pour l'homme. Certains solvants halogénés sont également classés comme cancérogènes certains ou probables pour l'homme.

14. La plupart des Hydrocarbures aromatiques polycycliques contiennent une substance considérée comme cancérogène certain pour l'homme.

15. Le GISCOP 93 retrace le parcours professionnel de patients malades d'un cancer potentiellement d'origine professionnelle pour identifier les expositions à des cancérogènes qu'ils ont subies. L'un des patients du groupement a travaillé 26 ans sur le site de Sevran, il a notamment été exposé à deux cancérogènes : des gaz d'échappement et à des révélateurs (produits utilisés dans l'industrie de la photographie). Il est décédé des suites d'un cancer broncho-pulmonaire.

16. Poly-exposition subie par 12 patients (source GISCOP 93).

17. Le texte évoque alors la renommée de l'activité ( renommée internationale notamment auprès des musées ») et la désertion du site est contrebalancée par le classement des fours au titre de Monuments Historiques.

18. La localisation de ces infrastructures se trouve sur le site web de la commune et sur le parcours de visite du patrimoine industriel de la commune.

\section{RÉSUMÉS}

L'histoire industrielle de la Seine-Saint-Denis a fortement marqué son territoire. Pourtant, ses traces, matérielles et immatérielles, sont menacées par les transformations urbaines, économiques, sociales et politiques du département et avec elles la mémoire des conditions sanitaires de travail. Le présent article analyse la visibilité d'une dégradation de la santé - 
immédiate ou plus tardive - des travailleurs et des riverains au regard des choix d'aménagement des anciens sites industriels. Dans un contexte où la Seine-Saint-Denis est déjà porteuse de nombreuses représentations négatives, nous constaterons que le « recyclage urbain » de ces sites tend à les valoriser (ou à valoriser le devenir du terrain) contribuant à rendre invisibles les conséquences sanitaires des activités productives - passées et présentes - dans le département.

The significant industrialization of Seine-Saint-Denis has left a strong mark on its territory. However, its tangible and intangible marks are threatened. The urban, economic, social and political transformations have progressively affected the industrial history and the memory of sanitary work conditions. This paper is aimed at analysing the visibility of industry's health impacts on workers and residents compared to the recognition of industrial heritage. In a context where the Seine-Saint-Denis region is already associated with many negative perceptions, we will focus on the "urban recycling" of these sites which contributes to render invisible the health consequences of industrial activities.

\section{INDEX}

Mots-clés : industrie, patrimoine industriel, mémoire, recyclage urbain, santé, Seine-SaintDenis

Keywords : industrial plants, industrial heritage, memory, process of recycling urban space, health, Seine-Saint-Denis

\section{AUTEURS}

\section{AXELLE CROISÉ}

Axelle Croisé, Axelle.Croise@etu.univ-paris1.fr, est doctorante en géographie, UMR 8586 PRODIG, et associée au Groupement d'Intérêt Scientifique sur les Cancers d'Origine Professionnelle en Seine-Saint-Denis (GISCOP 93). Elle a récemment publié :

- Lysaniuk B., Croisé A., Tabeaud M., Counil E., 2015. La difficile compilation d'information(s) géographique(s) relative(s) à un cancérogène : étude pilote sur les expositions professionnelles à l'amiante en Seine-Saint-Denis. Pollution atmosphérique, n² 225, p. 1-16.

\section{ALEXIS SIERRA}

Alexis Sierra, a.alexis.sierra@gmail.com, est maître de Conférence HDR en Géographie, à l'Université de Cergy-Pontoise-ESPE et membre de l'UMR 8586 PRODIG. Il a publié récemment : - Delaitre M., Sierra A., Barroca B., Vargas Florez J., 2018. De la lecture d'une crise à celle de la résilience territoriale : le phénomène el Niño2017 au Pérou vu depuis la capitale. Risques urbains, vol. 2, $\mathrm{n}^{\circ}$ 1, p. 1-27.

- Sierra A., 2017. Paroxysmes, saisir la ville par la rupture. In Rapport Prospective Nationale de la Recherche Urbaine, Paris, CNRS.

- Jacquot S., Sierra A., Tadié J. 2016. Informalité politique, pouvoirs et envers des espaces urbains. L'Espace Politique [En ligne], nº 29. http://journals.openedition.org/espacepolitique/3805

\section{BENJAMIN LYSANIUK}

Benjamin Lysaniuk, Benjamin.Lysaniuk@cnrs.fr, est chargé de recherche à l'IRD (mis à disposition par le CNRS), UMR 8586-215 PRODIG, et associé au Groupement d'Intérêt Scientifique sur les Cancers d'Origine Professionnelle en Seine-Saint-Denis (GISCOP 93). Il a publié 
récemment :

- Lysaniuk B., 2018. Le sens des cartes. In Gaille M. (dir.), Établir la preuve d'une pathologie environnementale. Enjeux épistémologiques et politiques. Paris, Editions du CNRS, p.113-136.

- Tabeaud M., Lysaniuk B., 2017. Céder à la pression. In Bost F. et al., Images économiques du monde 2018. France : la grande fracture. Paris, Armand Colin, p. 73-75. 